\title{
The expanding phenotype of stroke-induced immune alterations
}

\author{
Johanna Ruhnau, Antje Vogelgesang, Alexander Dressel \\ Department of Neurology, Neuroimmunology Section, University Medicine Greifswald, 17489 Greifswald, Germany.
}

Ruhnau J, Schulze K, Gaida B, Langner S, Kessler C, Broker B, Dressel A, Vogelgesang A. Stroke alters respiratory burst in neutrophils and monocytes. Stroke 2014;45(3):794-800.

\section{INTRODUCTION}

The clinical outcome of ischemic stroke patients is determined by the extent of the ischemic lesion as well as the occurrence of severe systemic infections. Patients with pneumonia have a three-fold increase in 30-day mortality, and survivors have poorer clinical outcomes. ${ }^{[1]}$

Pneumonia has long been known to complicate the clinical course of many stroke patients. It was assumed that being bedridden and the occurrence of dysphagia would account for the increased risk of stroke-associated infections (SAI). However, over the last years, it has been demonstrated that stroke-induced immune alterations (SIIA) observed in patients and experimental stroke models are strong predictors of subsequent poststroke infections. ${ }^{[2-4]}$ Furthermore, one study associated insular stroke localization with pneumonia. ${ }^{[5]}$

These findings led to a new concept in which SIIA are causally related to SAI. Here, we briefly outline the current knowledge on SIIA and SAI and highlight recent studies that investigated cell functions involved in early bacterial defense mechanisms.

\begin{tabular}{|l|l|}
\hline \multicolumn{2}{|c|}{ Access this article online } \\
\hline Quick Response Code: & \\
\hline & Website: \\
\hline & Www.nnjournal.net \\
& \\
\hline
\end{tabular}

\section{STROKE-INDUCED IMMUNE ALTERATIONS}

Stroke-induced immune alterations can be observed in the peripheral blood of stroke patients within the first hour after stroke. Blood samples obtained shortly after stroke already exhibit severe lymphocytopenia. Stress hormones are thought to be closely involved in these rapid changes; stress hormone levels have been shown to be elevated in human stroke patients, blockade of catecholamines prevents SAI in animal models of experimental stroke, and in vitro exposure of peripheral blood mononuclear cell to stress hormones mimics some aspects of SIIA. ${ }^{[6,7]}$ Our initial observation in humans that lymphopenia was associated with susceptibility to infection was subsequently confirmed by several groups. ${ }^{[3,8]}$

It was unexpected that these disease induced immune alterations which reduced lymphocyte counts and induced granulocytosis should account for enhanced susceptibility to infection because the first line of defense against these infections is the innate immune response. In humans, the functional deactivation of monocytes (e.g. reduced human leukocyte antigen expression, reduced release of tumor necrosis factor- $\alpha$ ) was detected after cerebral ischemia and was shown to be associated with a higher risk of poststroke infections. ${ }^{[3,9]}$ We investigated in detail bactericidal mechanisms of monocytes and granulocytes after stroke.

Granulocytes home to the site of infection and kill pathogens by degranulation and phagocytosis, via a process termed oxidative burst and the release of web-like structures called neutrophil extracellular traps (NETs). During inflammation and infection, granulocytes and monocytes are recruited by chemoattractants and inflammatory cytokines. The cells adhere and internalize pathogens by endocytosis. Due to the generation of toxic

Corresponding Author: Prof. Alexander Dressel, Department of Neurology, University Medicine Greifswald, Sauerbruchstr., 17489 Greifswald, Germany. E-mail: adressel@uni-greifswald.de 
radicals, they are able to eliminate pathogens by oxidative burst. In addition, neutrophils can release bactericidal peptides (e.g. $\alpha$-defensin human neutrophil peptides 1-3 [HNP 1-3]) to fight against extracellular pathogens. Another killing strategy of neutrophils is the release of NETs. These complexes of decondensed chromatin consist of over 30 different neutrophil proteins that can capture, neutralize, or eliminate different pathogens. These structures form a physical barrier to reduce the spread of pathogens and enhance the concentrations of antimicrobial effectors. ${ }^{[10-12]}$

We recently investigated these granulocyte and monocyte functions in 63 human stroke patients. Our data demonstrated that migration, phagocytosis, and the release of HNP 1-3 were unimpaired in the peripheral blood of stroke patients compared with healthy controls. However, oxidative burst and NET formation were impaired in stroke patients. Our observations are in line with a previous small study that indicated an impaired oxidative burst in 17 patients with hemorrhagic stroke. ${ }^{[13]}$ Furthermore, cells obtained from patients that went on to develop SAI showed a stronger impairment of oxidative burst capacity than patients without subsequent infections. These data suggest that alterations in innate immune functions may be causally related to SAI susceptibility. Alterations in lymphocyte counts in the peripheral blood of stroke patients may indicate SIIA severity, without directly affecting the host's bacterial defense capacity.

\section{REFERENCES}

1. Katzan IL, Cebul RD, Husak SH, Dawson NV, Baker DW. The effect of pneumonia on mortality among patients hospitalized for acute stroke. Neurology 2003;60:620-5.
2. Chamorro A, Urra X, Planas AM. Infection after acute ischemic stroke: a manifestation of brain-induced immunodepression. Stroke 2007;38:1097-103.

3. Vogelgesang A, Grunwald U, Langner S, Jack R, Bröker BM, Kessler C, Dressel A. Analysis of lymphocyte subsets in patients with stroke and their influence on infection after stroke. Stroke 2008;39:237-41.

4. Vogelgesang A, Dressel A. Immunological consequences of ischemic stroke: immunosuppression and autoimmunity. J Neuroimmunol 2011;231:105-10.

5. Steinhagen V, Grossmann A, Benecke R, Walter U. Swallowing disturbance pattern relates to brain lesion location in acute stroke patients. Stroke 2009;40:1903-6.

6. Harms H, Reimnitz P, Bohner G, Werich T, Klingebiel R, Meisel C, Meisel A. Influence of stroke localization on autonomic activation, immunodepression, and post-stroke infection. Cerebrovasc Dis 2011;32:552-60.

7. Katan M, Elkind MS. Inflammatory and neuroendocrine biomarkers of prognosis after ischemic stroke. Expert Rev Neurother 2011;11:225-39.

8. Hug A, Dalpke A, Wieczorek N, Giese T, Lorenz A, Auffarth G, Liesz A, Veltkamp R. Infarct volume is a major determiner of post-stroke immune cell function and susceptibility to infection. Stroke 2009; 40:3226-32.

9. Prass K, Meisel C, Höflich C, Braun J, Halle E, Wolf T, Ruscher K, Victorov IV, Priller J, Dirnagl U, Volk HD, Meisel A. Stroke-induced immunodeficiency promotes spontaneous bacterial infections and is mediated by sympathetic activation reversal by poststroke T helper cell type 1-like immunostimulation. J Exp Med 2003;198:725-36.

10. Brinkmann V, Zychlinsky A. Beneficial suicide: why neutrophils die to make NETs. Nat Rev Microbiol 2007;5:577-82.

11. Papayannopoulos V, Zychlinsky A. NETs: a new strategy for using old weapons. Trends Immunol 2009;30:513-21.

12. Mesa MA, Vasquez G. NETosis. Autoimmune Dis 2013;2013:651497.

13. Seki Y, Sahara Y, Itoh E, Kawamura T. Suppressed neutrophil respiratory burst in patients with haemorrhagic stroke. J Clin Neurosci 2010;17:187-90.

Cite this article as: Ruhnau J, Vogelgesang A, Dressel A. The expanding phenotype of stroke-induced immune alterations. Neuroimmunol Neuroinflammation 2014;1(1):15-6.

Source of Support: Nil. Conflict of Interest: No.

Received: 09-05-2014; Accepted: 30-05-2014 